\title{
A Branching Process Approach to Power Markets
}

\author{
Ying Jiao* Chunhua $\mathrm{Ma}^{\dagger} \quad$ Simone Scotti ${ }^{\ddagger} \quad$ Carlo Sgarra $^{\S}$
}

February 28, 2018

\begin{abstract}
We propose and investigate a market model for power prices, including most basic features exhibited by previous models and taking into account self-exciting properties. The model proposed extends Hawkes-type models by introducing a two-fold integral representation property. A Random Field approach was already exploited by BarndorffNielsen et al., who adopted the Ambit Field framework for describing the power price dynamics. The novelty contained in our approach consists in combining the basic features of both Branching Processes and Random Fields in order to get a realistic and parsimonious model setting. We shall provide some closed-form evaluation formulae for forward contracts. We discuss the risk premium behavior, by pointing out that in the present framework, a very realistic description arises. We outline a possible methodology for parameters estimation. We illustrate by graphical representation the main achievements of this approach.
\end{abstract}

Keywords: Branching Processes, Energy Markets, Self-Exciting Structures, Risk Premium Term Structure, Random Fields.

\section{Introduction}

Energy markets, and in particular, electricity markets, exhibit very peculiar features. The historical series of both futures and spot prices include seasonality, mean-reversion, spikes and small fluctuations.

After the pioneering paper by Schwartz [34, where an Ornstein-Uhlenbeck dynamics is assumed to describe the spot price behavior, several different approaches have been investigated in order to describe the price evolution. A comprehensive presentation of

\footnotetext{
*Université Claude Bernard-Lyon 1, Institut de Science Financier et d'Assurances. Email: ying.jiao@univ-lyon1.fr.

${ }^{\dagger}$ Nankai University, School of Mathematical Sciences. Eamil: mach@nankai.edu.cn.

${ }^{\ddagger}$ Université Paris Diderot-Paris 7, Laboratoire de Probabilités et Modèles Aléatoires. Email: scotti@math.univ-paris-diderot.fr.

${ }^{\S}$ Politecnico di Milano, Dipartimento di Matematica. Email: carlo.sgarra@polimi.it
} 
the literature until 2008 is offered in the book by F.E. Benth, J. Salthythe-Benth and S. Koekebakker [7].

High frequency trading, on the other hand, introduced some new features in commodity prices dynamics: in the paper by V. Filimonov, D. Bicchetti, N. Maystre and D. Sornette 21] evidence is shown of endogeneity and structural regime shift, and in order to quantify this level the branching ratio is adopted as a measure of this endogenous impact and a Hawkes processes dynamics is assumed as a reasonable modeling framework taking into account the self-exciting properties [1].

Among the several different approaches proposed in the literature in order to describe the spiking phenomenon characterizing the power price dynamics we mention the paper by C. Lucheroni [32], where two alternative models based on Stochastic FitzHugh-Nagumo system are introduced. By exploiting the stochastic resonating properties of the solutions in a suitable regime, the author is able to explain several basic features of the power market behavior. Moreover, a systematic investigation performed on the Alberta Power Market data from April 7, 2002 and April 6, 2007, exhibits a very good fitting of the model to real data; in particular, the interspike distribution described by both models seems to reproduce very well the spike frequency observed.

V. Barbu, F. Cordoni and L. Di Persio [16], and F. Cordoni, L. Di Persio [17] provided recently some results on an optimal control problem for the stochastic FitHugh-Nagumo model, pointing out the impossibility to apply standard optimal control methods due to the cubic nonlinearity of the system and making the financial applicability of this model quite challenging, especially considering that some of the most extensively traded options on energy markets, that is swing options, belong, roughly speaking, to the class of American options.

In contrast with the papers just cited, the main focus of the present work is on the jump clustering phenomenon, which is another puzzling feature exhibited by spiking patterns. The purpose of the present paper is to propose a new modeling framework including all the above mentioned features, still keeping a high level of tractability. The model considered allows to obtain the most common derivatives prices in closed or semi-closed form. Here with semi-closed we mean that the Laplace transform of the derivative price admits an explicit expression.

R. Kiesel and F. Paraschiv [29] presented recently a systematic empirical investigation of electricity intraday prices and suggested an approach based on Hawkes processes in order to describe the power price dynamics and the jump clustering feature. Self-exciting features in electricity prices attracted already some attention by several authors: although the above mentioned paper by V. Filimonov, D. Bicchetti, N. Maystre and D. Sornette [21] did not investigate endogenous effects in power markets, R. Herrera and N. Gonzalez [24] proposed a self-excited model for electricity spot prices, while T.M. Christensen, A.S. Hurn and K.A. Lindsay [11], Christensen, and A. Clements, J. Fuller and A.S. Hurn [12] pointed out that time between spikes has a significant impact on the likelihood of future occurrences, thus providing a strong support to models including self-exciting properties.

The models we are going to introduce can describe the price dynamics in two different forms, that can be proved to be equivalent: the first is a representation based on random 
fields, the second is based on Continuous State Branching Processes with Immigration (CBI in the following). The idea of adopting a random fields framework for power prices description is not new: O.E. Barndorff-Nielsen, F.E. Benth and A. Veraart introduced the Ambit Fields to this end, showing how this approach can provide a very flexible and still tractable setting for derivatives pricing [3], 4]. Moreover CBI processes exhibit the Markov property, while Hawkes processes enjoy this property only in particular cases, and this feature makes our approach more appealing.

A model based on CBI has been proposed recently by Y. Jiao, C. Ma and S. Scotti in view of short interest rate modeling, and in that paper it was shown that, with a suitable choice of the Lévy process driving the CBI dynamics, the model can offer a significant extension of the popular CIR model [25].

The model we propose extends in different ways some relevant models already available in the literature. It belongs to the class of arithmetic models (following the classification proposed by F.E. Benth, J. Salthythe-Benth and S. Koekebakker), and the driving processes are Lévy processes with positive jumps, i.e. subordinators, so it extends the model proposed by F.E. Benth, J. Kallsen and T. Meyer-Brandis [5] by formulating the dynamics via a random field approach, which allows to include some self-exciting features. On the other hand, the random field approach highlights some similarities with the Ambit Field-based models introduced by O.E. Barnorff-Nielsen, F.E. Benth and A. Veraart [4]; the main difference between the model proposed in this paper and the Ambit Field-based models consists in the character of the extra dimension appearing in the random field adopted: while in the Ambit Field setting the parameter of this dimension is a time parameter, in the present setting this will be a parameter of space type. This main difference will be reflected moreover in the integration domain of the integrals defining the dynamics.

The features of our modeling approach just outlined, allow to introduce the so-called self-exciting properties in a simple and natural way and, although the pricing formulas for basic contracts like forward will exhibit very small changes with respect to those obtained for the previous models, the present model will exhibit a substantially different risk premium term structure.

The paper is organized as follows: in Section 2 we introduce the market model we are going to consider, while in Section 3 we discuss the relations between our model and the CBI processes. In Section 4 we derive some closed formulas for Futures and Option prices when the underlying dynamics is assumed to be given by the model introduced. Section 5 includes a theoretical analysis of the jumps behavior and the self-exciting property. In Section 6 we provide some evidence of clustering behavior of power prices based on real data. In the final section we provide some concluding remarks and discuss futures extensions of the present work.

\section{The Modeling Framework}

\subsection{Model setup}

We now introduce our modeling framework for the electricity price, which is based on stochastic differential equations driven by Lévy random field. We consider a Lévy random 
field which is a combination of a Gaussian random measure $W$ and a compensated Poisson random measure $N$ independent to $W$. For background for such general stochastic equations with jumps, we refer the readers to Dawson and Li [15], Li and Ma [31] and Walsh [35].

Let us briefly present the preliminaries. We fix a probability space $(\Omega, \mathcal{A}, \mathbb{P})$. A white noise $W$ on $\mathbb{R}_{+}^{2}$ is a Gaussian random measure such that, for any Borel set $A \in \mathcal{B}\left(\mathbb{R}_{+}^{2}\right)$ with finite Lebesgue measure $|A|, W(A)$ is a normal random variable of mean zero and variance $|A|$; and that if $A_{1}, \cdots, A_{n}$ are disjoint Borel sets in $\mathcal{B}\left(\mathbb{R}_{+}^{2}\right)$, then $W\left(A_{1}\right), \cdots, W\left(A_{n}\right)$ are mutually independent. We denote by $N$ the Poisson random measure on $\mathbb{R}_{+}^{3}$ with intensity $\lambda$ which is a Borel measure on $\mathbb{R}_{+}^{3}$ in form of the product of the Lebesgue measure on $\mathbb{R}_{+} \times \mathbb{R}_{+}$with a Borel measure $\mu$ on $\mathbb{R}^{+}$such that $\int_{0}^{\infty}\left(\zeta \wedge \zeta^{2}\right) \mu(d \zeta)<+\infty$. Note that $\mu$ is a Lévy measure since $\int_{0}^{\infty}\left(1 \wedge \zeta^{2}\right) \mu(d \zeta)<+\infty$. Recall that for each Borel set $A \in \mathcal{B}\left(\mathbb{R}_{+}^{3}\right)$ with $\lambda(A)<+\infty$, the random variable $N(A)$ has the Poisson distribution with parameter $\lambda(A)$; moreover, if $A_{1}, \ldots, A_{n}$ are disjoint Borel sets in $\mathcal{B}\left(\mathbb{R}_{+}^{3}\right)$, then $N\left(B_{1}\right), \cdots, N\left(B_{n}\right)$ are mutually independent. We let $\widetilde{N}=N-\lambda$ be the compensated Poisson random measure on $\mathbb{R}_{+}^{3}$ associated to $N$.

We introduce the filtration $\mathbb{F}=\left(\mathcal{F}_{t}\right)_{t \geqslant 0}$ as the natural filtration generated by the Lévy random field and satisfying the usual conditions, namely, for any Borel subset $A \in \mathcal{B}\left(\mathbb{R}_{+}\right)$ and $B \in \mathcal{B}\left(\mathbb{R}_{+}^{2}\right)$ of finite Lebesgue measure, the processes $(W([0, t] \times A), t \geq 0)$ and $(\widetilde{N}([0, t] \times B), t \geq 0)$ are $\mathbb{F}$-martingales.

We consider the following stochastic differential equation in the integral form.

Definition 2.1. Let $a, b, \sigma, \gamma \in \mathbb{R}_{+}$be constant parameters. Consider the equation

$Y(t)=Y(0)+\int_{0}^{t} a(b-Y(s)) d s+\sigma \int_{0}^{t} \int_{0}^{Y(s)} W(d s, d u)+\gamma \int_{0}^{t} \int_{0}^{Y(s-)} \int_{\mathbb{R}^{+}} \zeta \tilde{N}(d s, d u, d \zeta)$

where $W(d s, d u)$ is a white noise on $\mathbb{R}_{+}^{2}$ with unit covariance, $\tilde{N}(d s, d u, d \zeta)$ is an independent compensated Poisson random measure on $\mathbb{R}_{+}^{3}$ with intensity $\lambda=d s d u \mu(d \zeta)$ with $\mu(d \zeta)$ being a Lévy measure on $\mathbb{R}_{+}$and satisfying $\int_{0}^{\infty}\left(\zeta \wedge \zeta^{2}\right) \mu(d \zeta)<\infty$.

The integral appearing in the previous formula (and in the following) is in the sense of [35]. It follows from [15, Theorem 3.1] or [31, Theorem 2.1] that the equation (2.1) has a unique strong solution. Below are several particular examples.

Example 2.1. (CIR model) In the case where $\gamma=0$, we have

$$
\begin{aligned}
Y(t) & =Y(0)+\int_{0}^{t} a(b-Y(s)) d s+\sigma \int_{0}^{t} \int_{0}^{Y(s)} W(d s, d u) \\
& =Y^{*}(t)+\sigma \int_{0}^{t} \int_{0}^{Y(s)} e^{-a(t-s)} W(d s, d u),
\end{aligned}
$$

where $Y^{*}(t)=r_{0} e^{-a t}+a b \int_{0}^{t} e^{-a(t-s)} d s$ is a deterministic function. This corresponds to the classic CIR model in the interest rate where an equivalent representation of (2.2) in probability is given as

$$
Y(t)=Y(0)+\int_{0}^{t} a(b-Y(s)) d s+\sigma \int_{0}^{t} \sqrt{Y(s)} d B(s)
$$

where $B=(B(t), t \geq 0)$ is a Brownian motion. 
Example 2.2. ( $\alpha$-CIR model) As a generalization of the CIR model with jumps, let $\sigma=0$ and the Lévy measure $\mu$ be given by as

$$
\mu(d \zeta)=-\frac{1_{\{\zeta>0\}} d \zeta}{\cos (\pi \alpha / 2) \Gamma(-\alpha) \zeta^{1+\alpha}}, \quad 1<\alpha<2,
$$

then the model (2.1) admits the following representation

$$
Y(t)=Y(0)+\int_{0}^{t} a(b-Y(s)) d s+\gamma \int_{0}^{t}(Y(s-))^{\frac{1}{\alpha}} d L(s)
$$

where $L=(L(t), t \geq 0)$ is a spectrally positive compensated $\alpha$-stable Lévy process with parameter $\alpha \in(1,2]$, which is independent of $B$ and whose Laplace transform is given, for $q \geq 0$, by

$$
\mathbb{E}\left[e^{-q L(t)}\right]=\exp \left(-\frac{t q^{\alpha}}{\cos (\pi \alpha / 2)}\right) .
$$

When $\alpha=2$, L reduces to a Brownian motion scaled by $\sqrt{2}$ and we recover the standard CIR model. This model is studied in [25] for short interest rate.

\subsection{Model comparison}

In the literature on energy modeling, both random fields and Lévy processes have been adopted to describe electricity prices such as in Barndorff-Nielsen, Benth and Veraart [3, 4]. In [4], ambit fields have been used to model future prices and in [3], the so-called volatility modulated Lévy-driven Volterra (VMLV) processes have used to model spot prices. Both approaches provide tractable results and allow to capture stylized facts observed on energy markets.

We can compare our model to the ambit fields model introduced in [3, 4]. From the mathematical point of view, both formulations exhibit a random field setting where one more integrated variable appears with respect to classical stochastic evolution models. The main difference between the two approaches is that in [4] the variable is of time type whereas in our case is of space type. This difference in turn implies a different integration domain, that in our case depends on the process itself. Then, the main financial difference here is that the model (2.1) exhibits explicitly the self-exciting property which is suitable to describe the clustering effect in the power price dynamics. We explain in more detail the self-exciting feature of our model and start by considering the following example.

Example 2.3. Let $\sigma=0$ and $\mu(d \zeta)=\delta_{1}(d \zeta)$, then the (non-compensated) Poisson measure $N(d s, d u, d \zeta)$ reduces to a random measure on $\mathbb{R}_{+}^{2}$ with intensity dsdu, denoted by $N(d s, d u)$. Hence $Y$ can be rewritten as

$$
Y(t)=Y(0)+a b t-\int_{0}^{t}\left(a+\sigma_{N}\right) Y(s) d s+\gamma \int_{0}^{t} \int_{0}^{Y(s-)} N(d s, d u) .
$$

We note that $Y$ is the intensity of the Hawkes process $\int_{0}^{t} \int_{0}^{Y(s-)} N(d s, d u)$ by using the equivalent form

$$
Y(t)=Y(0) e^{-(a+\gamma) t}+\frac{a b}{a+\gamma}\left(1-e^{(a+\gamma) t}\right)+\gamma \int_{0}^{t} \int_{0}^{Y(s-)} e^{-(a+\gamma)(t-s)} N(d s, d u) .
$$


More precisely, let us consider a simple Hawkes process $J$ with exponential kernel with the intensity given by:

$$
Y(t)=Y^{*}(t)+\gamma \int_{0}^{t} e^{-a(t-s)} d J(s)
$$

where $Y^{*}$ is the background rate, i.e., the deterministic part of the process $J$. When a jump arrives, the intensity increases, which also increases the probability of a next jump, that is the self-exciting property of Hawkes processes. In order to facilitate the comparison with our integral representation, we give an alternative characterization of the intensity. Let $N$ be a Poisson process on $\mathbb{R}_{+}^{2}$ with measure $d s d u$, so $J(t)$ can be written as $\int_{0}^{t} \int_{0}^{Y(s-)} N(d s, d u)$ and $Y(t)$ as $Y^{*}(t)+\gamma \int_{0}^{t} \int_{0}^{Y(s-)} e^{-a(t-s)} N(d s, d u)$. In this form, the self-exciting feature can be observed more explicitly: the frequency of jumps grows with the process itself due to the presence of the integral with respect to the variable $u$. Therefore we note from the expressions (2.2) and 2.5 the self-exciting property inherited from our model framework.

Remark 2.1. By focusing on the last example, we can better illustrate the relation between the Hawkes-based approach and our framework. In the last example the jump measure is just a Dirac measure. By considering a more general Poisson measure, we can obtain again the process (2.1) by simply introducing a mark $\zeta$. The process defined by 2.1) then can be interpreted as a "marked" Hawkes process.

Moreover, when $Y^{*}$ takes certain particular form, $Y$ becomes a branching process, also known as the affine process in finance (see Duffie, Filipović and Schachermayer [18] and Filipović [22]). In this context, the self-exciting features is equivalent to the branching property and the jump intensity is proportional to the process $Y$ itself. Then the models we have introduced can describe the prices dynamics in two ways: the first is the random field formulation exhibiting explicitly the clustering behavior, the second is the Continuous Branching processes with Immigration (CBI) structure, that will be presented in the next subsection.

\subsection{Connection with CBI and affine processes}

The purpose of this section is to highlight the relation between the process class introduced in the previous section and the classes of CBI processes and affine processes. This relation will turn out to be relevant in order to apply transform-based methods to derivatives evaluation. Our model actually belongs to the family of CBI processes. We briefly recall the definition by Kawazu and Watanabe [28]. A Markov process $X$ with state space $\mathbb{R}_{+}$ is called a CBI process characterized by branching mechanism $\Psi(\cdot)$ and immigration rate $\Phi(\cdot)$, if its characteristic representation is given, for $p \geq 0$, by

$$
\mathbb{E}_{x}\left[e^{-p X(t)}\right]=\exp \left(-x v(t, p)-\int_{0}^{t} \Phi(v(s, p)) d s\right)
$$

where the function $v: \mathbb{R}_{+} \times \mathbb{R}_{+} \rightarrow \mathbb{R}_{+}$satisfies the following differential equation

$$
\frac{\partial v(t, p)}{\partial t}=-\Psi(v(t, p)), \quad v(0, p)=p
$$


and $\Psi$ and $\Phi$ are functions of the variable $q \geq 0$ given by

$$
\begin{array}{r}
\Psi(q)=\beta q+\frac{1}{2} \sigma^{2} q^{2}+\int_{0}^{\infty}\left(e^{-q u}-1+q u\right) \pi(d u), \\
\Phi(q)=\delta q+\int_{0}^{\infty}\left(1-e^{-q u}\right) \nu(d u),
\end{array}
$$

with $\sigma, \gamma \geq 0, \beta \in \mathbb{R}$ and $\pi, \nu$ being two Lévy measures such that

$$
\int_{0}^{\infty}\left(u \wedge u^{2}\right) \pi(d u)<\infty, \quad \int_{0}^{\infty}(1 \wedge u) \nu(d u)<\infty .
$$

It is proved in Dawson and Li [15, Theorem 3.1] that the process (2.1) is a CBI process with the branching mechanism $\Psi$ given by

$$
\Psi(q)=a q+\frac{1}{2} \sigma^{2} q^{2}+\int_{0}^{\infty}\left(e^{-q \sigma_{Z} \zeta}-1+q \sigma_{Z} \zeta\right) \mu(d \zeta)
$$

and the immigration rate $\Phi(q)=a b q$.

The link between CBI processes and the affine interest rate term structure models has been established by Filipović [22]. If the process takes values in $\mathbb{R}_{+}$he proves equivalence between the two classes. We recall the joint Laplace transform of a CBI process $X$ and its integrated process, which is given in [22, Theorem 5.3], as follows. For non-negative real numbers $\xi$ and $\theta$, we have

$$
\mathbb{E}_{x}\left[e^{-\xi X(t)-\theta \int_{0}^{t} X(s) d s}\right]=\exp \left\{-x v(t, \xi, \theta)-\int_{0}^{t} \Phi(v(s, \xi, \theta)) d s\right\}
$$

where $v(t, \xi, \theta)$ is the unique solution of

$$
\frac{\partial v(t, \xi, \theta)}{\partial t}=-\Psi(v(t, \xi, \theta))+\theta, \quad v(0, \xi, \theta)=\xi
$$

Remark 2.2. We just proved that for the present model class an explicit expression of the moment generating function is available, from which all moments of the density distribution can be computed, as long as they exist and are finite. These moments can be used, in principle, as a statistical tool for inference. An extensive investigation on statistical properties of the present class of models and a rigorous discussion of possible estimation methods will be anyway presented in a forthcoming paper.

\section{$3 \quad$ Modeling the electricity price}

In this section, we apply the previous modeling framework to the description of electricity price dynamics. We follow the arithmetic model as in [3, 5, 6] in which the power price dynamics is assumed to be the sum of several factors which follow for example VMLV processes in [3] and non-Gaussian Ornstein-Uhlenbeck processes in [5, 6]. In our model the factors are assumed to be given by the SDE (2.1). 


\subsection{An arithmetic model for the spot price}

We assume that the spot price process $S=(S(t), t \geq 0)$ evolves according to the following dynamics

$$
S(t)=\alpha(t)+X(t)
$$

where $\alpha(t)$ is a deterministic function representing the seasonality, and the process $X=$ $(X(t), t \geq 0)$ is a superposition of the factors $Y_{i}$ as

$$
X(t)=\sum_{i=1}^{n} Y_{i}(t) .
$$

The factors $Y_{i}(t)$ evolve according to the equation 2.1, more precisely,

$$
\begin{aligned}
Y_{i}(t)=Y_{i}(0) & +\int_{0}^{t} a_{i}\left(b_{i}-Y_{i}(s)\right) d s+\sigma_{i} \int_{0}^{t} \int_{0}^{Y_{i}(s)} W_{i}(d s, d u) \\
& +\gamma_{i} \int_{0}^{t} \int_{0}^{Y_{i}(s-)} \int_{\mathbb{R}^{+}} \zeta \widetilde{N}_{i}(d s, d u, d \zeta)
\end{aligned}
$$

where $W_{i}(d s, d u)$ are independent Gaussian random measures and $\widetilde{N}_{i}(d s, d u, d \zeta)$ are independent compensated Poisson measures with compensators $d s d u \mu_{i}(d \zeta)$ as defined in Definition 2.1. The factor model described by (3.15) extends naturally the one proposed in 5] by keeping the basic features of an Ornstein-Uhlenbeck process driven by a subordinator but introducing the self-exciting properties in a direct way.

In the case where $\int_{\mathbb{R}_{+}} \zeta \mu_{i}(d \zeta)<\infty$, we can separate the Poisson random measure $N(d s, d u, d \zeta)$ from its predictable compensator $d s d u \mu_{i}(d \zeta)$. One typical example is the Pareto distribution with

$$
\mu_{i}(\xi>z)=\left(\frac{\max \left\{z, z_{0}\right\}}{z_{0}}\right)^{-\alpha}, \quad 1<\alpha<2,
$$

which has been studied in Meyer-Brandis and Tankov [33. In this case, we can describe the dynamics 3.15 as follows:

$$
\begin{aligned}
Y_{i}(t)=Y_{i}(0)-\int_{0}^{t} a_{i}\left(b_{i}-Y_{i}(s)\right) d s+\sigma_{i} \int_{0}^{t} \int_{0}^{Y_{i}(s)} W_{i}(d s, d u) \\
\quad-\gamma_{i} \int_{0}^{t} d s \int_{0}^{Y_{i}(s-)} d u \int_{\mathbb{R}^{+}} \zeta \mu_{i}(d \zeta)+\gamma_{i} \int_{0}^{t} \int_{0}^{Y_{i}(s-)} \int_{\mathbb{R}^{+}} \zeta N_{i}(d s, d u, d \zeta)
\end{aligned}
$$

which is equivalent to

$$
\begin{aligned}
Y_{i}(t)=Y_{i}(0) & -A_{i} \int_{0}^{t}\left(B_{i}-Y_{i}(s)\right) d s+\sigma_{i} \int_{0}^{t} \int_{0}^{Y_{i}(s)} W_{i}(d s, d u) \\
& +\gamma_{i} \int_{0}^{t} \int_{0}^{Y_{i}(s-)} \int_{\mathbb{R}^{+}} \zeta N_{i}(d s, d u, d \zeta),
\end{aligned}
$$

where

$$
A_{i}:=a_{i}-\gamma_{i} \int_{\mathbb{R}^{+}} \zeta \mu_{i}(d \zeta), \quad B_{i}:=\frac{a_{i} b_{i}}{A_{i}}
$$


The expression (3.16) shows how a different mean-reversion speed for each factor $Y_{i}$ can arise even if a common mean-reversion speed is assigned in the form (2.1).

Below two trajectories are plotted of the spot price in a two-factor model where the first factor $Y_{1}$ follows a standard CIR model as in Example 2.1 and the second factor $Y_{2}$ is an $\alpha$-CIR process as in Example 2.2. For simplicity of illustration, we let the seasonal deterministic function $\alpha(t)=0$. We observe the clustering effect for large jumps.

In Figure 1 the trajectory is plotted with parameters $Y_{1}(0)=30, a_{1}=3, b_{1}=30$, $\sigma_{1}=3$, the second factor includes just the jump contribution with $Y_{2}(0)=35 a_{2}=5$, $b_{2}=35, \gamma_{2}=5$ and the tail parameter $\alpha=1.5$.

In order to get a more faithful description of the power prices dynamics it is convenient to increase the values of the parameters, in agreement with the values proposed in the previous literature, see [6], in particular the mean reversion speed and the jumps sizes should be magnified. In Figure 2 the trajectory is plotted with same parameters as in the previous case except $Y_{1}(0)=Y_{2}(0)=5, a_{1}=10, a_{2}=20, b_{1}=b_{2}=5, \gamma_{2}=10$, exhibiting a more realistic behavior.

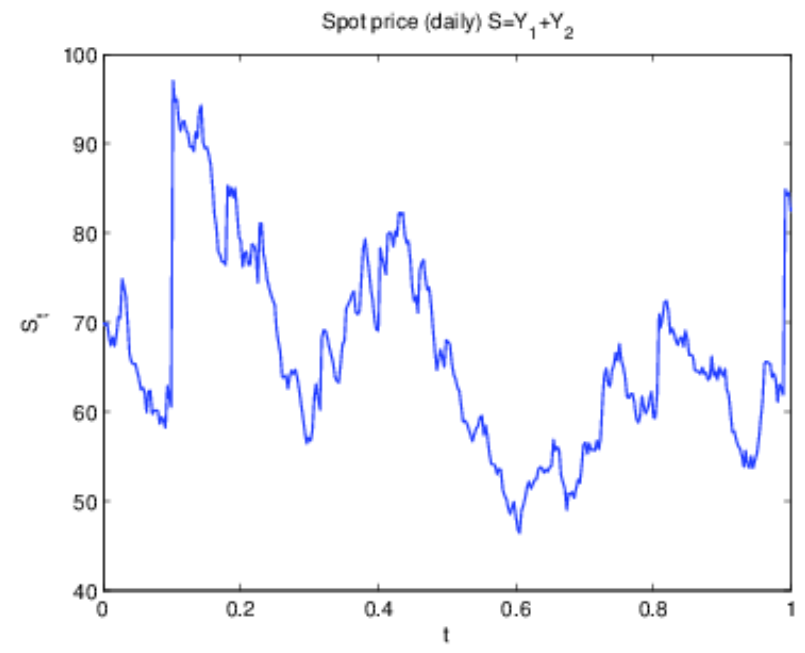

Figure 1: Small values for the mean reversion speed and jumps size.

The following result computes the expected value of the factors $Y_{i}$ given in (3.15) and will be useful for the pricing of energy derivatives in the next section.

Lemma 3.1. For any $T \geq 0$ and $\tau \leq T$, we have

$$
\mathbb{E}\left[Y_{i}(T) \mid \mathcal{F}_{\tau}\right]=b_{i}+\left(Y_{i}(\tau)-b_{i}\right) e^{-a_{i}(T-\tau)} .
$$

Proof. First of all we have

$$
\mathbb{E}\left[Y_{i}(T)\right]=Y_{i}(0)+\mathbb{E}\left[\int_{0}^{T} a_{i}\left(b_{i}-Y_{i}(s)\right) d s\right] .
$$

Then, thanks to the uniqueness of solution of (3.15), denoting $\phi(t):=\mathbb{E}\left[Y_{i}(t)\right]$ and applying Fubini's theorem we obtain the following ordinary differential equation

$$
\frac{\partial}{\partial t} \phi(t)=a_{i}\left(b_{i}-\phi(t)\right) \text {. }
$$




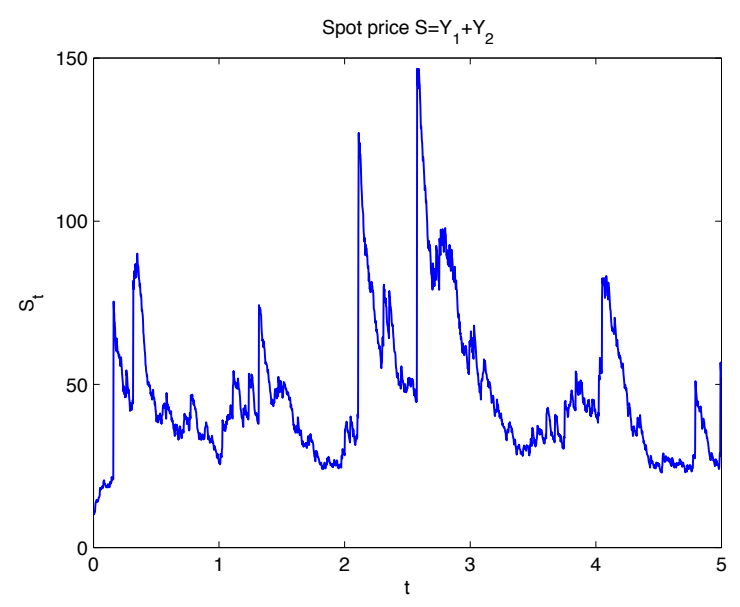

Figure 2: Simulation of the spot price in a two-factor model with magnified parameters.

The solution is then

$$
\mathbb{E}\left[Y_{i}(t)\right]=b_{i}+\left(Y_{i}(0)-b_{i}\right) e^{-a_{i} t} .
$$

By a direct application of Markov property we obtain (3.17).

Remark 3.3. In the model just proposed all the jumps are assumed to be positive. Due to renewable energy production negative jumps can be also observed in power prices. Negative jumps could also be considered in the present modelling framework, but the introduction of negative jumps breaks out some of the properties related to the moment generating functions existence. CBI with negative jumps have been considered in [2] in order to model cell infection dynamics. Negative jumps can be introduced also in an indirect way by considering factors of the same form proposed here, but with a negative sign. In a two factor model this would mean $X(t)=Y_{1}(t)-Y_{2}(t)$. This choice would preserve all the basic properties of the model, but the existence of a common domain of definition for the moment generating function of both driving factors is a delicate issue that must be carefully considered for each jump structure proposed.

\section{Evaluation of energy derivatives}

In this section, we investigate how to evaluate derivatives in our modeling framework for power dynamics. We first explain how to choose a risk-neutral probability $\mathbb{Q}$ in the model setup described in the previous section. We then discuss the pricing issues together with the risk premium in the electricity market.

\subsection{Change of probability measure}

In the electricity market, the risk-neutral probability $\mathbb{Q}$ is often chosen by introducing a drift adjustment in the dynamics of an underlying asset. Compared to the original historical probability $\mathbb{P}$, such measure change is done by using Esscher transform such as in [3, 4]. We now define the equivalent probability measures in our model. The following proposition 
shows that the spot process $S$ defined in $(3.13)$ will remain in the same class, that is, the factors $Y_{i}$ are still CBI processes but with modified coefficients under a suitable equivalent change of probability. The proof is based on the CBI characterization for the factors defined in (2.1).

Proposition 4.1. Let $Y_{1}, Y_{2}, \cdots, Y_{n}$ be independent $C B I$ processes where for each $i \in$ $\{1, \cdots, n\}, Y_{i}$ is a $C B I$ process under the probability measure $\mathbb{P}$. Assume that the filtration $\mathbb{F}=\left(\mathcal{F}_{t}\right)_{t \geq 0}$ is generated by the random fields $W_{1}, W_{2}, \cdots, W_{n}$ and $\widetilde{N}_{1}, \widetilde{N}_{2}, \cdots, \widetilde{N}_{n}$. For each $i$, fix $\eta_{i} \in \mathbb{R}$ and $\theta_{i} \in \mathbb{R}_{+}$and define

$$
U_{t}:=\sum_{i=1}^{n} \eta_{i} \int_{0}^{t} \int_{0}^{Y_{i}(s)} W_{i}(d s, d u)+\sum_{i=1}^{n} \int_{0}^{t} \int_{0}^{Y_{i}(s-)} \int_{0}^{\infty}\left(e^{-\theta_{i} \zeta}-1\right) \widetilde{N}_{i}(d s, d u, d \zeta) .
$$

Then the Doléans-Dade exponential $\mathcal{E}(U)$ is a martingale and the probability measure $\mathbb{Q}$ defined by

$$
\left.\frac{d \mathbb{Q}}{d \mathbb{P}}\right|_{\mathcal{F}_{t}}=\mathcal{E}(U)_{t},
$$

is equivalent to $\mathbb{P}$. Moreover, under $\mathbb{Q}$, the processes $Y_{1}, Y_{2}, \cdots Y_{n}$ are independent of each other and, for each $i, Y_{i}$ is a CBI process with the parameters $\left(a_{i}^{\prime}, b_{i}^{\prime}, \sigma_{i}^{\prime}, \gamma_{i}^{\prime}, \mu_{i}^{\prime}\right)$, where

$$
\begin{gathered}
a_{i}^{\prime}=a_{i}-\sigma_{i} \eta_{i}-\gamma_{i} \int_{0}^{\infty} \zeta\left(e^{-\theta_{i} \zeta}-1\right) \mu_{i}(d \zeta), \quad b_{i}^{\prime}=a_{i} b_{i} / a_{i}^{\prime}, \quad \sigma_{i}^{\prime}=\sigma_{i}, \quad \gamma_{i}^{\prime}=\gamma_{i} \\
\mu_{i}^{\prime}(d \zeta)=e^{-\theta_{i} \zeta} \mu_{i}(d \zeta) .
\end{gathered}
$$

Proof. The process $\left(Y_{1}, Y_{2}, \cdots, Y_{n}, U\right)$ is a time homogeneous affine process (c.f. [18, Theorem 2.12]). The Doléans-Dade exponential $\mathcal{E}(U)$ is a true martingale by checking that the conditions in [27, Corollary 3.2] are satisfied, so it defines an equivalent probability measure $\mathbb{Q}$. Note that $Z=\mathcal{E}(U)$ is the unique strong solution of $d Z_{t}=Z_{t-} d U_{t}$. Then for any function $F \in C^{2}\left(\mathbb{R}_{+}^{n}\right)$, the process

$$
\begin{aligned}
& Z_{t} F\left(Y_{1}(t), \cdots, Y_{n}(t)\right)-\sum_{i=1}^{n} \frac{\sigma_{i}^{2}}{2} \int_{0}^{t} Z_{s} F_{i i}^{\prime \prime}\left(Y_{1}(s), \cdots, Y_{n}(t)\right) Y_{i}(s) d s \\
& -\sum_{i=1}^{n} \int_{0}^{t} Z_{s} F_{i}^{\prime}\left(Y_{1}(s), \cdots, Y_{n}(s)\right)\left\{a_{i} b_{i}-\left[a_{i}-\sigma_{i} \eta_{i}-\gamma_{i} \int_{0}^{\infty} \zeta\left(e^{-\theta_{i} \zeta}-1\right) \mu_{i}(d \zeta)\right] Y_{i}(s)\right\} d s \\
& -\sum_{i=1}^{n} \int_{0}^{t} Z_{s} Y_{i}(s) d s \int_{0}^{\infty}\left[F\left(Y_{1}(s-), \cdots, Y_{i}(s-)+\gamma_{i} \zeta, \cdots, Y_{n}(s-)\right)\right. \\
& \left.\quad-F\left(Y_{1}(s-), \cdots, Y_{i}(s-), \cdots, Y_{n}(s-)\right)-F_{i}^{\prime}\left(Y_{1}(s-), \cdots, Y_{n}(s-)\right) \gamma_{i} \zeta\right] e^{-\theta_{i} \zeta} \mu_{i}(d \zeta)
\end{aligned}
$$

is a local martingale, which implies that under $\mathbb{Q}, Y_{1}, Y_{2}, \cdots Y_{n}$ are independent of each other and, for each $i, Y_{i}$ is a CBI process with the parameters $\left(a_{i}^{\prime}, b_{i}^{\prime}, \sigma_{i}^{\prime}, \gamma_{i}^{\prime}, \mu_{i}^{\prime}\right)$.

Usually we choose $\eta_{i}$ and $\theta_{i}$ such that $a_{i}^{\prime}>0$. When $\theta_{i}=0, \mu_{i}^{\prime}$ coincides with $\mu_{i}$, so that the CBI process $Y_{i}$ will remain in the same class under an equivalent change of probability measure. When $\theta_{i}>0, Y_{i}$ will become a new CBI process driven by another Lévy process with Lévy measure related by an exponential tilting to the original one. 


\subsection{Forward Pricing}

By applying the results presented in the previous sections, we can provide an explicit and simple expression for the (instantaneous) forward contract price in the present modeling framework. Recalling the definition of a forward contract, we have for any $\tau \leq T$ that

$$
F(\tau, T):=\mathbb{E}^{\mathbb{Q}}\left[S(T) \mid \mathcal{F}_{\tau}\right] .
$$

In the following of the present section, the model parameters are assumed to be those defined by the historical dynamics, in such a way that $a_{i}, b_{i}$ and $\mu_{i}$ will denote respectively the mean-reversion speed, the long-term value and the jump measure with respect to $\mathbb{P}$. Then we effectuate the equivalent change of probability measure as in Proposition 4.1 and the parameters $a_{i}^{\prime}, b_{i}^{\prime}$ and $\mu_{i}^{\prime}$ will be with respect to the probability $\mathbb{Q}$.

Since the factors $Y_{i}$ remain to be CBI processes under the probability change, the Lemma 3.1 can still be applied to deduce the forward price, as given in the following proposition.

Proposition 4.2. We have the following expression for the forward contract

$$
F(\tau, T)=S(\tau)+(\alpha(T)-\alpha(\tau))+\sum_{i=1}^{n}\left(e^{-a_{i}^{\prime}(T-\tau)}-1\right)\left(Y_{i}(\tau)-b_{i}^{\prime}\right)
$$

where $a_{i}^{\prime}$ and $b_{i}^{\prime}$ are as in 4.20.

Proof. By 4.22 and applying Lemma 3.1 under the probability $\mathbb{Q}$, we have

$$
F(\tau, T)=\alpha(T)+\sum_{i=1}^{n} \mathbb{E}^{\mathbb{Q}}\left[Y_{i}(T) \mid \mathcal{F}_{\tau}\right]=\alpha(T)+\sum_{i=1}^{n} b_{i}^{\prime}+\left(Y_{i}(\tau)-b_{i}^{\prime}\right) e^{-\alpha_{i}^{\prime}(T-\tau)}
$$

and in particular,

$$
S(\tau)=F(\tau, \tau)=\alpha(\tau)+\sum_{i=1}^{n} b_{i}^{\prime}+\left(Y_{i}(\tau)-b_{i}^{\prime}\right)
$$

Hence

$F(\tau, T)=S(\tau)+F(\tau, T)-F(\tau, \tau)=\alpha(T)-\alpha(\tau)+S(\tau)+\sum_{i=1}^{n}\left(e^{-a_{i}^{\prime}(T-\tau)}-1\right)\left(Y_{i}(\tau)-b_{i}^{\prime}\right)$.

That concludes the proof.

From the above proposition, we note the Markovian property of the forward price $F(\tau, T)$, which depends on the instantaneous value of the spot price at the time $\tau$ and of the different factors .

\subsection{Flow Forward Pricing}

Forward contracts in electricity markets deliver the underlying commodity over a period rather than at a fixed time, and for this reason they are sometimes called flow forwards. The future price with delivery period $\left[T_{1}, T_{2}\right]$ is defined by

$$
F\left(\tau, T_{1}, T_{2}\right)=\frac{1}{T_{2}-T_{1}} \mathbb{E}^{\mathbb{Q}}\left[\int_{T_{1}}^{T_{2}} S(u) d u \mid \mathcal{F}_{t}\right]=\frac{1}{T_{2}-T_{1}} \int_{T_{1}}^{T_{2}} F(\tau, u) d u,
$$

which leads directly to the following result. 
Corollary 4.1. The value of the flow forward contract $F\left(\tau ; T_{1}, T_{2}\right)$ is given, for $\tau<T_{1}<$ $T_{2}$, by

$$
\begin{aligned}
F\left(\tau, T_{1}, T_{2}\right)=S(\tau) & +\left(\frac{1}{T_{2}-T_{1}} \int_{T_{1}}^{T_{2}} \alpha(u) d u-\alpha(\tau)\right) \\
& -\sum_{i=1}^{n}\left(1-\frac{e^{-a_{i}^{\prime}\left(T_{1}-\tau\right)}-e^{-a_{i}^{\prime}\left(T_{2}-\tau\right)}}{a_{i}^{\prime}\left(T_{2}-T_{1}\right)}\right)\left(Y_{i}(\tau)-b_{i}^{\prime}\right)
\end{aligned}
$$

By comparing our results with those obtained by Benth, Kallsen and Meyer-Brandis [5], we can remark that the expressions of the forward and the flow forward contracts are very similar. In fact, taking into account the linearity of the two contracts considered and the arithmetic feature of the model, the contribution of the martingale term (which differentiates the two models) vanishes. However, this property no longer holds for more complex contracts as soon as non-linearity arises. Moreover, we will see in the next subsection that the risk premium also allows to differentiate between the two frameworks with the measure change.

\subsection{The Risk Premium}

The risk premium is an important quantity in power markets which provides a link between the forward and expected spot prices. We present an explicit representation formula for this quantity in our modeling framework. The risk premium is defined as the difference between conditional expectations of the underlying price computed with respect to the risk-neutral measure $\mathbb{Q}$ and the historical measure $\mathbb{P}$ :

$$
R(\tau, T)=\mathbb{E}^{\mathbb{Q}}\left[S(T) \mid \mathcal{F}_{\tau}\right]-\mathbb{E}^{\mathbb{P}}\left[S(T) \mid \mathcal{F}_{\tau}\right]
$$

Since the factors $Y_{i}$ remain to be CBI processes under the equivalent measure change, according to Lemma 3.1 and Proposition 4.2, we have the following result.

Corollary 4.2. Let the risk-neutral probability $\mathbb{Q}$ be given as in Proposition 4.1. Then we have

$$
\begin{aligned}
R(\tau, T)= & \sum_{i=1}^{n} Y_{i}(\tau)\left(e^{-a_{i}^{\prime}(T-\tau)}-e^{-a_{i}(T-\tau)}\right) \\
& \quad-\sum_{i=1}^{n}\left[b_{i}^{\prime}\left(e^{-a_{i}^{\prime}(T-\tau)}-1\right)-b_{i}\left(e^{-a_{i}(T-\tau)}-1\right)\right]
\end{aligned}
$$

where the parameters $a_{i}^{\prime}, b_{i}^{\prime}$ are related to the parameters $a_{i}$ and $b_{i}$ by the relations (4.20).

A systematic investigation on the risk premium for power markets models based on Lévy-driven Ornstein-Uhlenbeck processes was presented in the paper by Benth and Sgarra [8], while the basic features of the risk premium for the most popular power market models was discussed in the paper by Benth, Kiesel and Nazarova [6].

We note a few basic features of the risk premium in our model obtained by the previous formula. The crucial parameter $\theta$ will play the major role in determining the risk premium 
sign, which, according to many empirical investigations performed in different power markets, can change suddenly, and induce a change between a contango to a backwardation regime. A suitable choice of $\theta$ can give rise to this sign change typical of electricity markets. The most relevant feature exhibited by the present modeling framework is that the very simple measure change introduced in the previous section implies a change in the mean speed reversion parameter. This feature is not feasible for Lévy-driven Ornstein-Uhlenbeck models as long as simple measure changes are considered. The Esscher transform for the Lévy driver implies a change in the long-term mean, but not in the mean-reversion speed; this in turn implies a risk premium term structure exhibiting sign change only for very special choice of the exponential tilting parameter $\theta$ (see Benth and Sgarra [8]).

Figure 3 presents the risk premium $R(\tau, T)$ given by (4.26) as a function of the time to maturity $T-\tau$. We consider again a two-factor model with the following parameters (specified under the historical measure): $Y_{1}(0)=0, Y_{2}(0)=5, a_{1}=10, a_{2}=2, b_{1}=3$, $b_{2}=3.5, \sigma_{1}=\gamma_{2}=10$, while the values of the parameters specifying the measure change are $\eta_{1}=1$ and $\theta_{2}=0.005$ respectively. Notably, we are interested in the impact of $\alpha$ which is a determinant parameter in the second factor $Y_{2}$ modelled by an $\alpha$-CIR process. We observe that for the three values of $\alpha$, there is a change of sign in the risk premium with positive values in short term and negative values in long term. In particular, small values of $\alpha$ implying more significant variations in the risk premium, especially in the short term. This is due to the fact that the parameter $\alpha$ is an inverse measure of heaviness of distribution tails. More as $\alpha$ close to 1, more likely that the large jumps appear with deeper compensation between the jumps, so more probable there will be clustering effect.

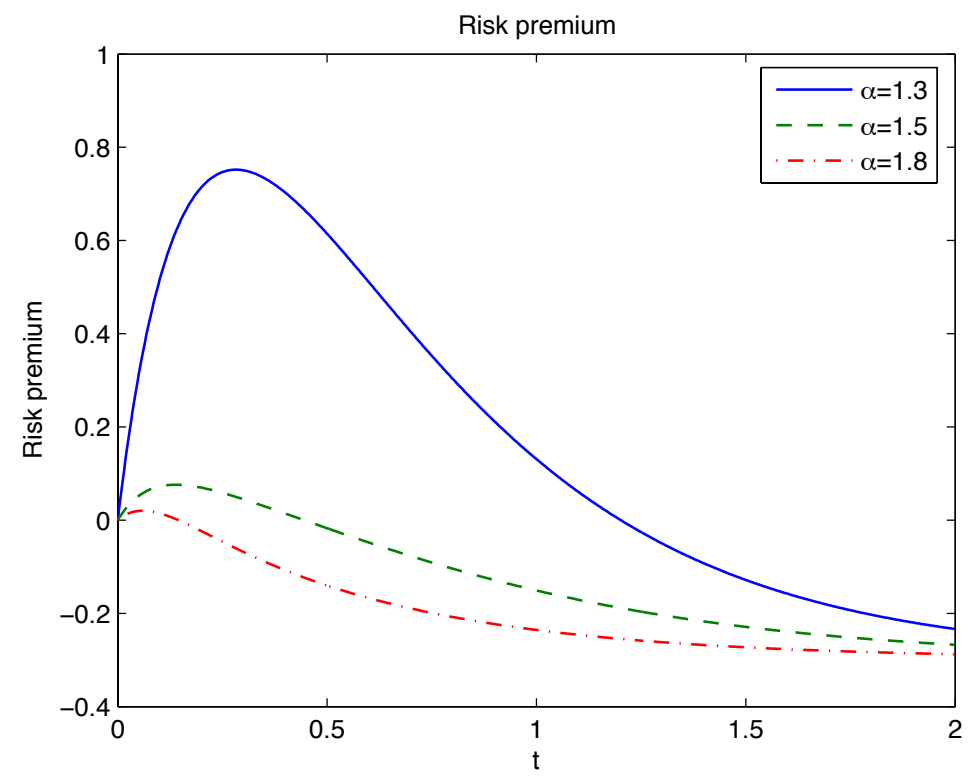

Figure 3: Risk premium term structure in a two factor model for different values of jump tail parameter $\alpha$. 


\subsection{Forward Option Pricing}

In the present modeling framework it is possible to obtain in an almost closed-form the prices of European options written on Forward contracts. We want to determine the noarbitrage value at time $t<\tau$ of a European call option with maturity $\tau$ and strike $K$ written on a forward contract with maturity $T$. The payoff of this option is $\max (F(\tau, T)-K, 0)$. Fourier and/or Laplace transform-based methods allow to obtain in closed form the value of the option considered if the underlying value (in this case the Forward value) transform is known in explicit form. We point out again that in all the present section the model parameters are assumed to be those defined by the risk-neutral dynamics, in such a way that $a_{i}, b_{i}$ and $\mu$ will denote the mean-reversion speed, the long-term value and the jump measure with respect to $\mathbb{Q}$. The formula obtained in the previous section providing the forward value, which we recall here for reading ease, is the following:

$$
F(\tau, T)=\alpha(T)-\alpha(\tau)+S(\tau)+\sum_{i}\left[e^{-a_{i}^{\prime}(T-\tau)}-1\right]\left[Y_{i}(\tau)-b_{i}^{\prime}\right] .
$$

By remembering the expression of $S(\tau)$, we can write the previous expression in a slighly modified form:

$$
F(\tau, T)=\alpha(T)+\sum_{i} Y_{i}(\tau)+\sum_{i}\left[e^{-a_{i}^{\prime}(T-\tau)}-1\right]\left[Y_{i}(\tau)-b_{i}^{\prime}\right] .
$$

For each of the terms appearing in the previous equation it is possible to compute the Laplace transform by applying Equation (2.7). Once this Laplace transform has been computed, the method proposed by P. Carr and D. Madan [10] or alternatively the method proposed by Lewis [30] allow to get the option price transform, from which the option price can be obtained by numerically inverting the transform considered. This last numerical step can be performed in an accurate and fast way.

As far as Flow Forward are concerned, it must be pointed out that one more integration step must be included in the computation according to Equation (4.24), but under suitable integrability conditions, it will be possible to exchange the integration sequence.

\section{Jumps Analysis in a Two-Factor Model}

In this section, we consider a model with two factors, which is similar as in Meyer-Brandis and Tankov [33. The first factor is continuous and corresponds to a standard CIR model and the second one is with jumps. Our objective is to make a thorough analysis of the jump behavior, in particular, for large jumps and spikes.

Let the first factor $Y_{1}$ be driven by a Gaussian random measure as

$$
Y_{1}(t)=Y_{1}(0)+\int_{0}^{t} a_{1}\left(b_{1}-Y_{1}(s)\right) d s+\sigma_{1} \int_{0}^{t} \int_{0}^{Y_{1}(s)} W_{1}(d s, d u), \quad t \geq 0
$$

and the second factor $Y_{2}$ be driven by a pure jump Poisson random measure as

$$
Y_{2}(t)=Y_{2}(0)+\int_{0}^{t} a_{2}\left(b_{2}-Y_{2}(s)\right) d s+\gamma_{2} \int_{0}^{t} \int_{0}^{Y_{2}(s-)} \int_{\mathbb{R}^{+}} \zeta \widetilde{N}_{2}(d s, d u, d \zeta), \quad t \geq 0 .
$$


The presence of large jumps and spikes is an important feature discussed in literature for electricity prices. A "spike" here means a sudden large rise in the price followed by a rapid drop to its regular level.

Remark 5.4. Comparing our model, given by equations (5.28) and (5.29), we can highlight that the main difference with respect to the model of Meyer-Brandis and Tankov is on the martingale part of (5.28) and (5.29) and in particular the integral until the process itself. The original model can then be obtained again suppressing this integral or freezing the time and obtaining a locally equivalent Lévy-Ornstein-Uhlenbeck as proposed in Definition 2.7 in [25].

In the following of this section, we focus on the jump factor $Y_{2}$ in order to analyze the spikes behavior in our model and to make a comparison with the previous models. In order to develop this analysis, it is necessary to get some theoretical intermediate results before focusing our interest on the statistical properties. In particular, we are interested in investigating the jumps frequency in order to validate the self-exciting features introduced [21]. We distinguish the contributions of different parts of the process $Y_{2}$, notably the large jumps and the remaining part which is regarded as a truncated process. We show below that apart from the large jumps, the truncated process will still behave as a CBI process but with a larger mean-reverting coefficient, which makes it go back more quickly to the standard level.

\section{$5.1 \quad$ Locally equivalent jump process}

The purpose of the present section is threefold: the first target is to introduce the spike frequency by identifying a suitable auxiliary process, the second is to prove formally that the last process is, up to a constant factor, the intensity of the next spike; the third is to show how $Y_{2}$ can be approximated by the intensity of a Hawkes-type process and this result will allow to adopt the maximum likelihood estimator for Hawkes processes as an approximate estimator for the model described. Consider a family of jump times $\left\{\tau_{k}\right\}_{k \in \mathbb{N}}$ of the process $Y_{2}$ which corresponds to significant movements of the market. For example, it can denote jump times larger than a threshold. More precisely, for a given constant $z_{0}$, we denote $\left\{\tau_{k}^{\left(z_{0}\right)}\right\}_{k \in \mathbb{N}}$ as the sequence of jump times of $Y_{2}$ whose jump sizes are equal or larger than $\gamma_{2} z_{0}$, i.e.,

$$
\tau_{k}^{\left(z_{0}\right)}=\inf \left\{t>\tau_{k-1}^{\left(z_{0}\right)}: \Delta Y_{2}(t)>\gamma_{2} z_{0}\right\}, \quad \tau_{0}^{\left(z_{0}\right)}=0 .
$$

In the following, we shall suppress the over-quote $z_{0}$ for sake of readability. We are interested in the evolution of process $Y_{2}$ between two jump times, that is for any $t \in\left[\tau_{k}, \tau_{k+1}\right)$,

$$
Y_{2}(t)=Y_{2}\left(\tau_{k}\right)+\int_{\tau_{k}}^{t} a_{2}\left(b_{2}-Y_{2}(s)\right) d s+\gamma_{2} \int_{\tau_{k}}^{t} \int_{0}^{Y_{2}(s-)} \int_{\mathbb{R}^{+}} \zeta \widetilde{N}_{2}(d s, d u, d \zeta) .
$$

With these notations, the time $\tau_{k+1}$ is the arrival time of the first jump after $\tau_{k}$ larger than $z_{0}$ for the measure $N_{2}$ or equivalently, larger than $\gamma_{2} z_{0}$ for $Y_{2}$. By the following result, we can separate small and large jumps and move the compensation inside the speed and mean coefficients. We remark that the second line of 5.32 describes a finite activity pure 
jump process, then it can be seen as a compounded Poisson process jumping only at an increasing sequence of times, such that only an almost surely finite number of jumps arrive in a compact set of time.

Proposition 5.3. The process $Y_{2}$ can be written, for all $t \in\left[\tau_{k}, \tau_{k+1}\right)$, as

$$
\begin{aligned}
Y_{2}(t)= & Y_{2}\left(\tau_{k}\right)+\int_{\tau_{k}}^{t} A_{2}\left(B_{2}-Y_{2}^{\left(z_{0}\right)}(s)\right) d s+\gamma_{2} \int_{\tau_{k}}^{t} \int_{0}^{Y_{2}^{\left(z_{0}\right)}(s-)} \int_{0}^{z_{0}} \zeta \widetilde{N}_{2}(d s, d u, d \zeta) \\
& +\gamma_{2} \int_{\tau_{k}}^{t} \int_{0}^{Y_{2}^{\left(z_{0}\right)}(s-)} \int_{z_{0}}^{\infty} \zeta N_{2}(d s, d u, d \zeta),
\end{aligned}
$$

where $z_{0}>0$ is a fixed constant,

$$
A_{2}:=a_{2}+\gamma_{2} \int_{z_{0}}^{\infty} \zeta \mu(d z), \quad B_{2}:=\frac{a_{2} b_{2}}{A_{2}}
$$

and $Y_{2}^{\left(z_{0}\right)}$ is the truncated process, for all $t \in\left[\tau_{k}, \tau_{k+1}\right)$, defined by

$$
Y_{2}^{\left(z_{0}\right)}(t)=Y_{2}\left(\tau_{k}\right)+\int_{\tau_{k}}^{t} A_{2}\left(B_{2}-Y_{2}^{\left(z_{0}\right)}(s)\right) d s+\gamma_{2} \int_{\tau_{k}}^{t} \int_{0}^{Y_{2}^{\left(z_{0}\right)}(s-)} \int_{0}^{z_{0}} \zeta \widetilde{N}_{2}(d s, d u, d \zeta)
$$

Proof. It follows from [14, Theorem 5.1 and Theorem 5.2] that (5.34) has a unique strong solution and $Y^{\left(z_{0}\right)}$ is a CBI process defined by 2.7 with

$$
\Psi_{z_{0}}(q)=A_{2} q+\int_{0}^{z_{0}}\left(e^{-q \gamma_{2} \zeta}-1+q \gamma_{2} \zeta\right) \mu(d \zeta) \quad \text { and } \quad \Phi_{z_{0}}(q)=A_{2} B_{2} q
$$

Now we turn to (5.29). Obviously,

$$
\begin{aligned}
Y_{2}(t)=Y_{2}\left(\tau_{k}\right) & +\int_{\tau_{k}}^{t} a_{2}\left(b_{2}-Y_{2}(s)\right) d s+\gamma_{2} \int_{\tau_{k}}^{t} \int_{0}^{Y_{2}(s-)} \int_{0}^{z_{0}} \zeta \widetilde{N}_{2}(d s, d u, d \zeta) \\
& +\gamma_{2} \int_{\tau_{k}}^{t} \int_{0}^{Y_{2}(s-)} \int_{z_{0}}^{\infty} \zeta \tilde{N}_{2}(d s, d u, d \zeta) .
\end{aligned}
$$

The above equation can be rewritten in the following form:

$$
\begin{aligned}
Y_{2}(t)= & Y_{2}\left(\tau_{k}\right)+\int_{\tau_{k}}^{t} A_{2}\left(B_{2}-Y_{2}(s)\right) d s+\gamma_{2} \int_{\tau_{k}}^{t} \int_{0}^{Y_{2}(s-)} \int_{0}^{z_{0}} \zeta \widetilde{N}_{2}(d s, d u, d \zeta) \\
& +\gamma_{2} \int_{\tau_{k}}^{t} \int_{0}^{Y_{2}(s-)} \int_{z_{0}}^{\infty} \zeta N_{2}(d s, d u, d \zeta) .
\end{aligned}
$$

Note that for $t \in\left[\tau_{k}, \tau_{k+1}\right)$ we have that $\int_{\tau_{k}}^{t} \int_{0}^{Y_{2}(s-)} \int_{z_{0}}^{\infty} \zeta N_{2}(d s, d u, d \zeta)=0$. Then, thanks to (5.35), $\left\{Y_{2}(t): t \in\left[\tau_{k}, \tau_{k+1}\right)\right\}$ satisfies (5.34). By the uniqueness of solutions of (5.34), we get that $\mathbb{P}\left(Y_{2}(t)=Y_{2}^{\left(z_{0}\right)}(t), \forall t \in\left[\tau_{k}, \tau_{k+1}\right)\right)=1$. Thus we have 5.32 . 
The main idea of Proposition 5.3 is to distinguish the original process $Y_{2}$ by the intensity of its jumps, that is, the truncated process $Y_{2}^{\left(z_{0}\right)}$ given by formula 5.32 .

These two processes coincide up to the next large jump occurred.

The process $Y_{2}^{\left(z_{0}\right)}$ is related to the intensity of large jumps since it appears inside the last integral in (5.32) associated to large jumps. The instantaneous intensity of the jumps of $Y_{2}$ at time $t$ is then $Y_{2}^{\left(z_{0}\right)}(t-)$ times the normalization term associated to the jump measure, i.e. $1_{\left\{\zeta>z_{0}\right\}} \mu(d \zeta)$.

We stated that the truncated process $Y_{2}^{\left(z_{0}\right)}$ is linked to the intensity of the large jumps of $Y_{2}$. The following proposition explains in detail this link. Up to a constant, the process $Y_{2}^{\left(z_{0}\right)}$ is the stochastic intensity or hazard rate of the random time of the next big jump.

Proposition 5.4. Let $\left\{\tau_{k}\right\}_{k \in \mathbb{N}}$ be defined by (5.30). Then we have

$$
\mathbb{P}\left(\tau_{k+1}-\tau_{k}>t\right)=\mathbb{E}\left[\exp \left\{-K_{Y}^{\left(z_{0}\right)} \int_{\tau_{k}}^{\tau_{k}+t} Y_{2}^{\left(z_{0}\right)}(s) d s\right\}\right]
$$

where the renormalisation term $K_{Y}^{\left(z_{0}\right)}=\gamma_{2} \int_{z_{0}}^{\infty} \zeta \mu_{2}(d \zeta)$ which is the proper truncated mass of the jumps distribution, and the frequency process $Y_{2}^{\left(z_{0}\right)}$ is given by (5.34).

Proof. Here we use the method given by [23]. By (5.32) of Proposition 5.3, we see that

$$
\left\{\tau_{k+1}-\tau_{k}>t\right\}=\left\{\int_{\tau_{k}}^{\tau_{k}+t} \int_{0}^{Y_{2}^{\left(z_{0}\right)}(s-)} \int_{z_{0}}^{\infty} \zeta N_{2}(d s, d u, d \zeta)=0\right\}
$$

Let $\mathcal{F}_{t}=\sigma\left\{Y_{2}(s): 0 \leq s \leq t\right\}$. Note that conditioned on $\mathcal{F}_{\tau_{k}},\left\{\int_{\tau_{k}}^{t} \int_{0}^{v} \int_{z_{0}}^{\infty} N(d s, d u, d \zeta)\right.$ : $\left.t \geq \tau_{k}, v \geq 0\right\}$ is independent of $\left\{Y_{2}^{\left(z_{0}\right)}(t): t \geq \tau_{k}\right\}$. Then

$$
\begin{aligned}
& \mathbb{P}\left(\tau_{k+1}-\tau_{k}>t \mid \mathcal{F}_{\tau_{k}} ; Y_{2}^{\left(z_{0}\right)}(s), s \geq \tau_{k}\right) \\
= & \mathbb{P}\left(\int_{\tau_{k}}^{\tau_{k}+t} \int_{0}^{Y_{2}^{\left(z_{0}\right)}(s-)} \int_{z_{0}}^{\infty} \zeta N_{2}(d s, d u, d \zeta)=0 \mid \mathcal{F}_{\tau_{k}} ; Y_{2}^{\left(z_{0}\right)}(s), s \geq \tau_{k}\right) \\
= & \exp \left\{-K_{Y}^{\left(z_{0}\right)} \int_{\tau_{k}}^{\tau_{k}+t} Y_{2}^{\left(z_{0}\right)}(s) d s\right\} .
\end{aligned}
$$

Finally, we deal with the asymptotic behaviour of $Y_{2}^{\left(z_{0}\right)}$ when the mean reverting speed $a_{2}$ diverges. Let us introduce the process $\widehat{Y}_{2}^{\left(z_{0}\right)}$ defined as

$$
\widehat{Y}_{2}^{\left(z_{0}\right)}(t)=b_{2}+e^{-A_{2} t}\left[Y_{2}(0)-b_{2}\right]+\gamma_{2} \int_{0}^{t} \int_{0}^{\widehat{Y}_{2}^{\left(z_{0}\right)}(s-)} \int_{z_{0}}^{\infty} e^{-A_{2}(t-s)} \zeta N_{2}(d s, d u, d \zeta) .
$$

Remark 5.5. The process $\widehat{Y}_{2}$ can be seen as the intensity of the marked Hawkes process $\int_{0}^{t} \int_{0}^{\widehat{Y}_{2}^{\left(z_{0}\right)}(s-)} \int_{z_{0}}^{\infty} \zeta N_{2}(d s, d u, d \zeta)$ with exponential kernel, see section 2.2 in [25].

The next proposition shows that the two processes $Y_{2}$ and $Y_{2}^{\left(z_{0}\right)}$ have the same behaviour when $a_{2}$ goes to infinity. As a consequence, we can approximate the frequency of large jumps by the one of the Hawkes process as soon as $a_{2}$ is large. Then, if $a_{2}$ is large enough, the intensity of the jumps exhibits two behaviours. It is quite stable around $b_{2}$ but it jumps at all jumps times $\left\{\tau_{k}\right\}_{k \in \mathbb{N}}$ and exhibits an fast exponential decay to $b_{2}$ with speed $A_{2} \geq a_{2}$. 
Proposition 5.5. Consider $Y_{2}$ with $\mathbb{E}\left[Y_{2}(0)\right]<\infty$. As $a_{2} \rightarrow+\infty$, we have that for each $t>0, Y_{2}(t)-\widehat{Y}_{2}(t)$ goes to zero in probability.

Proof. First-of-all note that $A_{2} \rightarrow+\infty$ as $a_{2} \rightarrow+\infty$. Moreover $B_{2}=\frac{a_{2} b_{2}}{A_{2}} \rightarrow b_{2}$ in the same limit. We have that

$$
\begin{aligned}
Y_{2}(t)= & Y_{2}(0)+\int_{0}^{t} A_{2}\left(B_{2}-Y_{2}(s)\right) d s+\gamma_{2} \int_{0}^{t} \int_{0}^{Y_{2}(s-)} \int_{0}^{z_{0}} \zeta \widetilde{N}_{2}(d s, d u, d \zeta) \\
& +\gamma_{2} \int_{0}^{t} \int_{0}^{Y_{2}(s-)} \int_{z_{0}}^{\infty} \zeta N_{2}(d s, d u, d \zeta) .
\end{aligned}
$$

By Itô's formula,

$$
\begin{aligned}
Y_{2}(t)= & e^{-A_{2} t} Y_{2}(0)+\int_{0}^{t} A_{2} B_{2} e^{-A_{2}(t-s)} d s+\gamma_{2} \int_{0}^{t} \int_{0}^{Y_{2}(s-)} \int_{0}^{z_{0}} e^{-A_{2}(t-s)} \zeta \widetilde{N}_{2}(d s, d u, d \zeta) \\
& +\gamma_{2} \int_{0}^{t} \int_{0}^{Y_{2}(s-)} \int_{z_{0}}^{\infty} e^{-A_{2}(t-s)} \zeta N_{2}(d s, d u, d \zeta) .
\end{aligned}
$$

Then we have

$$
\begin{aligned}
Y_{2}(t)-\widehat{Y}_{2}^{\left(z_{0}\right)}(t)= & \gamma_{2} \int_{0}^{t} \int_{0}^{Y_{2}(s-)} \int_{0}^{z_{0}} e^{-A_{2}(t-s)} \zeta \widetilde{N}_{2}(d s, d u, d \zeta) \\
& +\gamma_{2} \int_{0}^{t} \int_{\widehat{Y}_{2}^{\left(z_{0}\right)}(s-)}^{Y_{2}(s-)} \int_{z_{0}}^{\infty} e^{-A_{2}(t-s)} \zeta N_{2}(d s, d u, d \zeta) .
\end{aligned}
$$

Furthermore, we also have

$$
\begin{aligned}
& \mathbb{E}\left[\left(\int_{0}^{t} \int_{0}^{Y_{2}(s-)} \int_{0}^{z_{0}} e^{-A_{2}(t-s)} \zeta N_{2}(d s, d u, d \zeta)\right)^{2}\right] \\
& =\mathbb{E}\left[\int_{0}^{t} Y_{2}(s) e^{-2 A_{2}(t-s)} d s\right] \int_{0}^{z_{0}} \zeta^{2} \mu(d \zeta) \\
& \leq C_{1}\left(1-e^{-2 A_{2} t}\right) / A_{2},
\end{aligned}
$$

for some positive constants $C_{1}$ and

$$
\begin{aligned}
\mathbb{E}\left[\int_{0}^{t} \int_{\widehat{Y}_{2}^{\left(z_{0}\right)}(s-)}^{Y_{2}(s-)} \int_{z_{0}}^{\infty} e^{-A_{2}(t-s)} \zeta N_{2}(d s, d u, d \zeta)\right] \\
\leq \mathbb{E}\left[\int_{0}^{t} \int_{\widehat{Y}_{2}^{\left(z_{0}\right)}(s-)}^{Y_{2}(s-)} \mathbb{1}_{\left\{Y_{2}(s-) \geq Y_{2}^{\left(z_{0}\right)}(s-)\right\}} \int_{z_{0}}^{\infty} e^{-A_{2}(t-s)} \zeta N_{2}(d s, d u, d \zeta)\right] \\
+\mathbb{E}\left[\int_{0}^{t} \int_{Y_{2}(s-)}^{\left(z_{0}\right)(s-)} \mathbb{1}_{\left\{Y_{2}(s-)<Y_{2}^{\left(z_{0}\right)}(s-)\right\}} \int_{z_{0}}^{\infty} e^{-A_{2}(t-s)} \zeta N_{2}(d s, d u, d \zeta)\right] \\
\leq \mathbb{E}\left[\int_{0}^{t}\left|Y_{2}(s)-\widehat{Y}_{2}^{\left(z_{0}\right)}(s)\right| e^{-A_{2}(t-s)} d s\right] \int_{z_{0}}^{\infty} \zeta \mu(d \zeta) .
\end{aligned}
$$

A suitable modification of Gronwall inequality provides then the required result. 


\section{Jump Detection and Cluster Identification}

The goal of the present section is not to provide an extensive statistical analysis for the proposed model class, but to present some evidence based on real data enforcing the assumption of self-exciting and clustering behavior of jumps in power price dynamics. Given the observation of power price on Italian market we can perform a direct analysis and exhibit the presence of jumps. In the same spirit of Callegaro el al. 9], we identify jumps using an iterated re-weighted least squared technique. The daily data span the period between April 1, 2004 and December 31, 2015. We can identify 96 positive jumps, but their distribution is far from being uniform. Picture 4 shows this distribution (the yellow lines identify jumps times and sizes) and we easily observe the presence of clusters.

In order to reject the hypothesis of Poisson arrivals, i.e. jump with inter-temporal distance following an exponential law with constant intensity, we recall the following wellknown result, see for instance O. El Euch and M. Rosenbaum [19] page 13.

Proposition 6.6. Let $N$ be a non-homogeneous Poisson process with rate $\mu$. Denote by $T_{i}$ the increasing sequence of arrival times of the jumps of $N$. Then, fix $t>0$, conditionally on $N_{t}$, the vector $\left(T_{1} ; T_{2} ; \ldots ; T_{N_{t}}\right)$ has the same law as $\left(U_{(1)} ; U_{(2)} ;:: ; U_{(N t)}\right)$, i.e. the order statistics built from uniform IID random variables with density:

$$
\frac{\mu(s) I_{(s \in[0, t])}}{\int_{0}^{t} \mu(u) d u} .
$$

It is easy to see that if $\mu$ is constant, the inter-temporal jump arrival distance $T_{i}-T_{i-1}$ are uniformly distributed. We can then perform a Kolmogorov-Smirnov test by assuming a uniform distribution for inter-temporal jumps arrival times and this hypothesis is definitely rejected since the obtained value 1.82 is very large compared to the critical value for all usual rejection levels.

If we suppose now that the price process is purely self-exciting, that is the intensity of the jumps arrival is proportional to the process it-self, we can apply Proposition 6.6. The idea is to compare the time distribution of the spikes using the historical time and the cumulated price as a new time scale. To this end we introduce $\Lambda(t)$ is such that

$\Lambda(t)=\int_{0}^{t} P(s) d s$, where $P(s)$ is the electricity price itself. Then, the function $\Lambda$ is the cumulated value of the electricity price. By performing once more a Kolmogoroff-Smirnov test on the hypothesis of a self-excited Poisson distribution for power prices, we get the value 1.060 , which allows to not reject the hypothesis at level $\alpha=0.10$. Figure 5 illustrates the result of the goodness of fit: the small squares represent the historical values, while the small circles represent their scaled values. It can be remarked that the historical values (the small yellow diamonds) are not close to the ideal line in the central region of the graph, while the scaled values (the small blue diamonds) are much closer.

\section{Concluding Remarks}

In the present paper we proposed a new modeling framework for power price dynamics, combining all the basic features exhibited by power market, but including the jump clus- 
tering phenomenon. This modelling approach considered keeps a high level of tractability, in fact it allows to obtain the most common derivatives prices in closed or semi-closed form.

The models introduced can describe the prices dynamics in two different forms, that can be proved to be equivalent: the first is a representation based on random fields, the second is based on Continuous State Branching Processes with Immigration.

The model we proposed extends in different ways some relevant models already available in the literature. It belongs to the class of arithmetic models (following the classification proposed by F.E. Benth, J. Salthythe-Benth and S. Koekebakker), and the driving processes are Lévy processes with positive jumps, i.e. subordinators, so it extends the model proposed by F.E. Benth, J. Kallsen and T. Meyer-Brandis [5] by formulating the dynamics via a random field approach. On the other hand, this approach exhibits some similarities with the Ambit Field-based models introduced by O.E. Barnorff-Nielsen, F.E. Benth and A. Veraart [4].

In contrast with the approach presented in [32, where the spiking patterns of power prices are described in terms of a resonating regime in a FitzHugh-Nagumo stochastic dynamic framework, the main focus of this paper is on the jump clustering phenomenon and the self-exciting properties exhibited by CBI processes.

The features of our modeling approach just outlined, allow to introduce the so-called self-exciting properties in a simple and natural way and, although the pricing formulas for basic contracts like forward will exhibit very small changes with respect to those obtained for the previous models, the present model exhibits a substantially different, and much more realistic risk premium term structure.

The analysis proposed on real data in Section 6 strongly supports the model approach proposed.

The results illustrated in the present paper can be extended in several directions. Several financial contracts traded in power market do not admit closed form expressions in the present modelling framework and suitable numerical procedures must be proposed in order to evaluate these contracts in the present setting. Moreover, a rigorous and exhaustive statistical inference approach must be developed in order to allow a systematic investigation of different power markets and their description in terms of CBI.

\section{References}

[1] Bacry, E., Mastromatteo, J. and Muzy, J.-F. (2015) Hawkes Processes in Finance, Market Microstructure and Liquidity, 01(1), 1-59.

[2] Bansaye, V., Pardo Millan, J.C. and Smadi, C. (2013): On the extinction of continuous state branching processes with catastrophes, Electronic J. Probab., 18(106), 1-31.

[3] Barndorff-Nielsen, O.E., Benth, F.E. and Veraart, A. (2013): Modelling energy spot prices by volatility modulated Lévy driven Volterra processes, Bernoulli, 19, 803-845.

[4] Barndorff-Nielsen, O.E., Benth, F.E. and Veraart, A. (2014): Modelling Electricity Futures by Ambit Fields, Advances in Applied Probability, 46 (3), 719-745. 
[5] Benth, F. E., Kallsen J. and Meyer-Brandis T. (2007): A Non-Gaussian OrnsteinUhlenbeck Process for Electricity Spot Price Modeling and Derivatives Pricing, Appl. Math. Finance, 14(2), 153-169.

[6] Benth, F. E., Kiesel, R. and Nazarova A. (2012): A critical empirical study of three electricity price models, Energy Economics, 34, 1589-1616.

[7] Benth, F. E., Salthyte-Benth J. and Koekebakker S. (2008): Stochastic Modelling of Electricity and Related Markets, World Scientific, Singapore.

[8] Benth, F. E. and Sgarra C. (2012): The Risk Premium and the Esscher Transform in Power Markets, Stoch. Anal. Appl., 30(1), 20-43.

[9] Callegaro, G., Gaigi M., Scotti, S., and Sgarra C. (2017): Optimal investment in markets with over and under-reaction to information, Mathematics and Financial Economics, 11(3), 299-322.

[10] Carr, P. and Madan, D. Option valuation using the fast Fourier transform, Journal of Computational Finance, 2 (4), 61-73 (1998).

[11] Christensen, T.M., Hurn A.S. and Lindsay, K.A. (2009): It never rains, but it pours: modelling the persistence of spikes in electricity markets, Energy Journal 30, 25-48.

[12] Clements, A. , Fuller J. and Hurn, A.S. (2013): Semi-parametric forecasting of spikes in electricity prices, Economic Records 89, 508-521.

[13] Cox, J., Ingersoll, J. and Ross, S. (1985): A theory of the term structure of interest rate. Econometrica 53, 385-408.

[14] Dawson, D.A. and Li, Z. (2006): Skew convolution semigroups and affine Markov processes. Annals of Probabability, 34, 1103-1142.

[15] Dawson, D.A. and Li, Z. (2012): Stochastic equations, flows and measure-valued processes. Annals of Probability, 40(2), 813-857.

[16] Barbu, V., Cordoni, F. and Di Persio, L. (2015): Optimal control of stochastic Fitzhugh-Nagumo equation. Int. Journal of Control, 89(4), 746-756 .

[17] Cordoni, F. and Di Persio, L. (2017): Optimal control for the stochastic FitzhughNagumo model with recovery variable. ArXiV:1705.10227v1, 1-16.

[18] Duffie, D., Filipović, D. and Schachermayer, W. (2003): Affine processes and applications in finance, Annals of Applied Probability, 13(3), 984-1053.

[19] El Euch, O. and Rosenbaum, M. (2016): Perfect hedging in rough Heston Models, PREPRINT, ArXiv:1410.3394.

[20] Embrechts, P., Lininger T. and Lin, T. (2011): Multivariate Hawkes Process: an Application to Financial Data, Journal of Applied Probability, 48(A), 367-378. 
[21] Filimonov, V., Bicchetti, D., Maystre, N., Sornette, D. (2014): Quantification of the High Level of Endogeneity and Structural Regime Shifts in Commodity Markets, Journal of International Money and Finance, 42(C), 174-192.

[22] Filipović, D. (2001): A general characterization of one factor affine term structure models. Finance and Stochastics, 5, 389-412.

[23] He, X. and Li, Z. (2015): Distributions of jumps in a continuous-state branching process with immigration. Journal of Applied Probability, 53(4), 1166-1177.

[24] Herrera, R. and Gonzalez, N. (2014): The modeling and forecasting of extreme events in electricity spot markets. International Journal of Forecasting, 30, 477-490.

[25] Jiao, Y., Ma, C., Scotti, S. (2017): Alpha-CIR Model with Branching Processes in Sovereign Interest Rate Modelling, Finance and Stochastics, 21(3), 789-813.

[26] Jiao, Y., Ma, C., Scotti, S. and Zhou, C. (2018): Alpha-Heston Stochastic Volatility Model, PREPRINT.

[27] Kallsen, J. and Muhle-Karbe, J. (2010), Exponentially affine martingales, affine measure changes and exponential moments of affine processes, Stochastic Processes and their Applications, 120, 163-181.

[28] Kawazu, K. and Watanabe, S. (1971): Branching processes with immigration and related limit theorems. Theory Probab. Appl., 16, 36-54.

[29] Kiesel, R. and Paraschiv, F. (2016): Econometric Analusis of 15-minutes intraday electricity prices. SSRN-id2671379, 1-44.

[30] Lewis, A. (2001): Option Valuation under Stochastic Volatility. Finance Press, London.

[31] Li, Z. and Ma, C. (2015): Asymptotic properties of estimators in a stable Cox-IngersollRoss model. Stochastic Processes and their Applications, 125(8), 3196-3233.

[32] Lucheroni, C. (2007): Resonating models for the electric power market. Physical Review E, 76(5), 056116(1-13).

[33] Meyer-Brandis, T. and Tankov, P. (2008): Multi-factor jump-diffusion models of electricity prices. International Journal of Theoretical and Applied Finance, 11(5), 503-528.

[34] Schwarz, E.S. (1997): The stochastic behavior of commodity prices: Implications for valuation and hedging. Journal of Finance 52 (3), pp. 923-973.

[35] Walsh, J. (1980): An Introduction to Stochastic Partial Differential Equations. Ecole d' été de Probabilités de Saint-Flour XIV-1984, Lecture Notes in Mathematics 1180, 265-430. Springer, Berlin. 


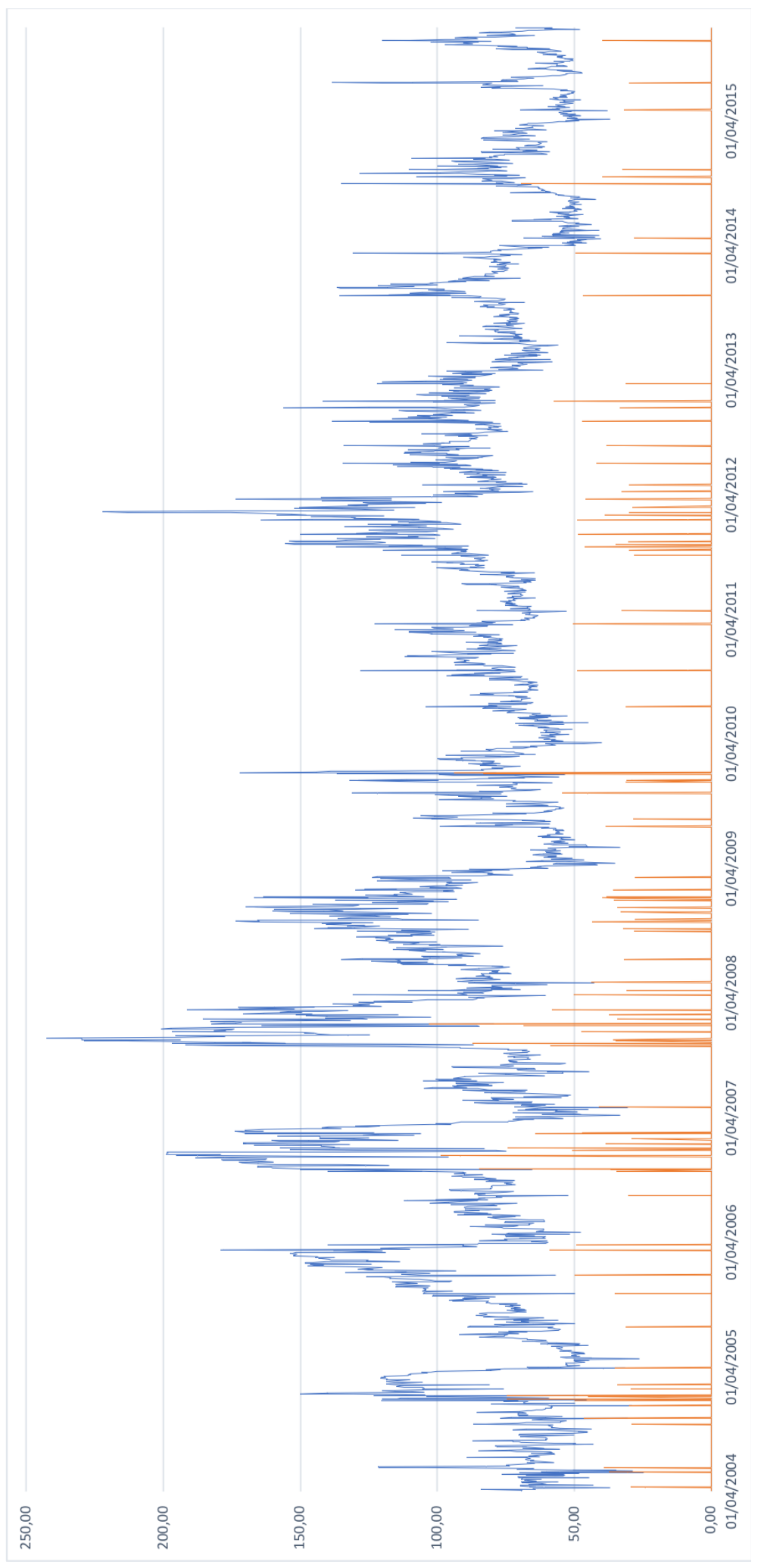

Figure 4: Historic time series of power price on Italian market from April 1, 2004 to December 31, 2015 


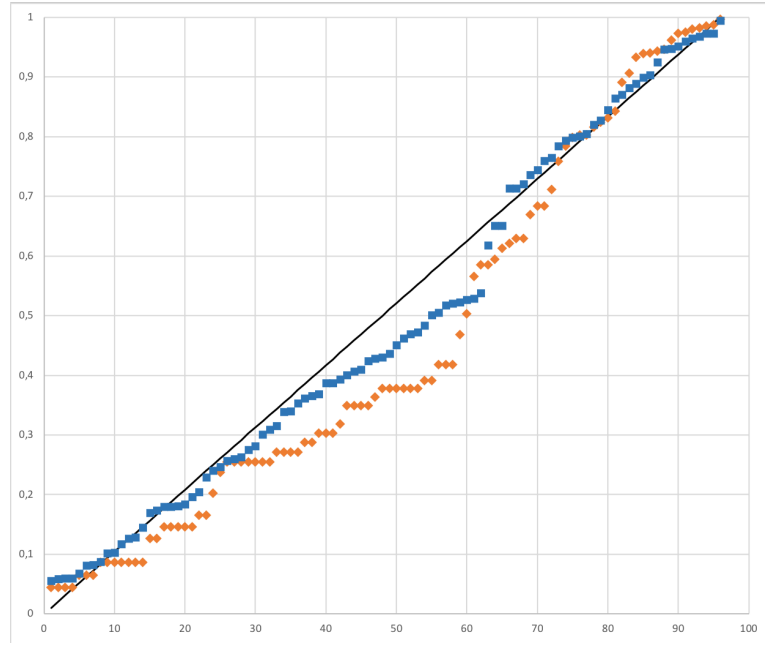

Figure 5: Kolmogorov-Smirnov test for self-excited Poisson hypothesis 